Review of:

Inhibition of in vivo breast cancer growth by antisense oligodeoxynucleotides to type I insulin-like growth factor receptor mRNA involves inactivation of ErbBs, PI-3K/Akt and p42/p44 MAPK signalling pathways but not modulation of progesterone receptor activity

\author{
A. V. Lee
}

The Breast Center, Baylor College of Medicine and the Methodist Hospital, Houston, TX, USA.

\title{
Citation of original article:
}

M. Salatino, R. Schillaci, C. J. Proietti, R. Carnevale, I. Frahm, A. A. Molinolo, A. Iribarren, E. H. Charreau, P. V. Elizalde. Oncogene 2004; 23(30): 5161-5174.

\begin{abstract}
of the original article
The present study addresses the effect of targeting type I insulin-like growth factor receptor (IGF-IR) with antisense strategies in in vivo growth of breast cancer cells. Our research was carried out on C4HD tumors from an experimental model of hormonal carcinogenesis in which the synthetic progestin medroxyprogesterone acetate (MPA) induced mammary adenocarcinomas in Balb/c mice. We employed two different experimental strategies. With the first one we demonstrated that direct intratumor injection of phosphorothioate antisense oligodeoxynucleotides (AS[S]ODNs) to IGF-IR mRNA resulted in a significant inhibition of C4HD tumor growth. In the second experimental strategy, we assessed the effect of intravenous (i.v.) injection of AS[S]ODN on C4HD tumor growth. This systemic treatment also resulted in significant reduction in tumor growth. The antitumor effect of IGF-IR AS[S]ODNs in both experimental protocols was due to a specific antisense mechanism, since growth inhibition was dose-dependent and no abrogation of tumor proliferation was observed in mice treated with phosphorothioate sense ODNs (S[S]ODNs). In addition, IGF-IR expression was inhibited in tumors from mice receiving AS[S]ODNs, as compared to tumors from control groups. We then investigated signal transduction pathways modulated in vivo by AS[S]ODNs treatment. Tumors from AS[S]ODN-treated mice of both intratumoral and i.v. protocols showed a significant decrease in the degree of insulin receptor substrate-1 (IRS-1) tyrosine phosphorylation. Activation of two of the main IGF-IR signaling pathways, phosphatidylinositol 3-kinase (PI-3K)/Akt and p42/p44 mitogen-activated protein kinases (MAPK) was abolished in tumors growing in AS[S]ODN-treated animals. Moreover, ErbB-2 tyrosine phosphorylation was blocked by in vivo administration of AS[S]ODNs. On the other hand, we found no regulation of either progesterone receptor expression or activity by in vivo AS[S]ODNs administration. Our results for the first time demonstrated that breast cancer growth can be inhibited by direct in vivo administration of IGF-IR AS[S]ODNs.
\end{abstract}

Correspondence to: Adrian V. Lee, The Breast Center, Baylor College of Medicine and the Methodist Hospital, Houston, TX, USA. E-mail: avlee@ breastcenter.tmc.edu; Tel +1713 798 1624; Fax: +1713 7981642

Publication date 28/01/05

BCO/328/2004/JC

\section{Review}

Insulin-like growth factors (IGFs) are critical regulators of normal cell growth. This has been shown in animal models and in human diseases, where disruption of 
IGF signalling results in dwarfism. However, as often happens in life, too much of a good thing can be bad. Thus, the potent proliferative and survival signals elicited by the IGFs are perfect for cancer growth and progression [1]. Indeed, it has been shown that IGF-I receptor (IGF-IR) is absolutely required for maintenance of the transformed phenotype [2]. The evidence that IGFs are key to tumour growth and progression has led to the IGF-IR becoming a major therapeutic target in cancer [3]. Several strategies to inhibit IGF-IR are in preclinical and clinical development, and the next 5 years will be pivotal in determining the applicability of anti-IGF strategies in cancer management and treatment.

Elizalde's group has been studying a mammary tumour (C4HD) that arose in synthetic progestin (methoxyprogesterone acetate (MPA)) treated Balb/c mice. The group has shown that this tumour is stimulated by MPA in vitro and in vivo [4]. This proliferation is driven by a complex interaction between progestins and growth factors. For example, MPA can upregulate levels of heregulin (HRG) and its receptor ErbB2 [5], and results in activation of an ErbB2/ ErbB3 complex. Blockade of HRG by antisense oligodeoxynucleotides (ASODN) abrogates the effect of MPA on C4HD cells, thus directly implicating HRG in the MPA-mediated proliferation. Interestingly, C4HD cells also express IGF-IR and secrete IGF-I and IGF-II, and ASODNs to IGF-II can also block MPA-induced proliferation [6].

A recent paper by Salatino et al. [7] describes the first application of antisense IGF-IR oligonucleotides to block mammary cancer growth in vivo. They showed that ASODN to IGF-IR efficiently block MPA-induced proliferation of C4HD cells in vitro, and that direct intratumor injection of ASODNs can also block tumour growth in vivo. They went on to show that intravenous (i.v.) administration of ASODNs can block the proliferation of C4HD tumours. This blockade of tumour growth was associated with a reduction in mitosis and the appearance of necrosis. Analysis of signalling within the tumours indicated that ASODN to IGF-IR caused a decrease in the levels and phosphorylation of IGF-IR, and that this was associated with decreased phosphorylation of insulin receptor substrate-1 (IRS-1), Akt and ERK1/2. They therefore concluded that the IGF-IR signalling pathway had been effectively blocked.

While the effect of i.v. ASODNs to IGF-IR further strengthens the rationale for IGF-IR as an excellent target for inhibition of breast cancer growth, one of the most intriguing aspects of this research is the apparent cross-talk between ErbB1/2 and IGF-IR. Several laboratories have noted bi-directional crosstalk between these receptors, with the term 'signal transactivation' appropriately describing this form of lateral signalling [8]. This was first described in IGF-IR null fibroblasts, which fail to respond to EGF and place IGF-IR as an upstream regulator of ErbB1 [9]. Similarly, Balana et al. [4] showed than in C4HD cells, ASODNs to IGF-IR blocked HRG action, and that there was a physical association between ErbB2 and IGF-IR. This same association was also found in MCF-7 human breast cancer cells and could be induced by either HRG or IGF-I. The authors summarized that there was a 'hierarchical interaction' between IGF-IR and HER-2 [4]. Salatino have now shown that this interaction can also be found in the C4HD tumours grown in vivo, and that ASODNs to IGF-IR can disrupt ErbB2/3 association and phosphorylation [7].

This is the first in vivo evidence that an anti-IGF-IR strategy can inhibit ErbB2/3 activity and clearly has important implications in breast cancer treatment. The interaction and bi-directional cross-talk between ErbB receptors and IGF-IR has been documented in a number of different cell systems (and now in vivo) and needs to be taken into account when considering either of these receptors as targets for blocking breast cancer progression. In this regard, it has already been shown that overexpression of IGF-IR can cause resistance to ErbB1 [10] or ErbB2 [11] inhibition. Given that IGF-IR seems to be an upstream regulator of ErbB action, this would make co-targeting of IGF-IR and ErbB1/2 an absolutely essential strategy for efficient ErbB1/2 blockade. In this regard, the recent reported bi-specific ErbB1/IGF-IR human monoclonal blocking antibody (ImClone Systems Incorporated, New York, NY) may be especially attractive [12].

Another important clinical aspect of the MPAinduced C4HD tumours is their progression to hormone independence. Similar to other models using human breast cancer cells, progression to hormone independence in $\mathrm{C} 4 \mathrm{HD}$ cells seems to involve a complex interaction between PR, IGF-IR, and EGFR/HER-2, with independence being associated with high constitutive levels of both HRG and IGF-II, and ASODNs to either of these ligands blocking the growth of C4HD cells $[5,6]$.

Several critical questions remain. First, what is the relevance of progestin-induced mammary cancer to human disease? Progesterone and its receptor (PR) remain a relatively understudied area in human breast cancer, often playing second fiddle to the oestrogen receptor (ER). However, recent hormone replacement therapy studies have clearly shown a role for progesterone in breast cancer, and recent clinical data regarding the utility of $P R$ in predicting antioestrogen resistance is starting to cause reappreciation of the role of progesterone and PR in breast cancer [13]. Second, is the PR cross-talk 
related or similar to ER cross-talk with growth factors? Can the last decade of research on cross-talk between ER and growth factors be used to rapidly elucidate cross-talk with PR? Third, are ASODNs viable therapeutic strategies for cancer treatment? Finally, what are the exact molecular mechanisms for cross-talk between ErbB1/2 and IGF-IR? Sophisticated biochemical analysis needs to be performed to understand how these receptors associate, and who is activating whom. Newly available IGF-IR and ErbB1/2 inhibitors will surely aid in this effort.

In summary, Salatino et al's report provides further exciting evidence that IGF-IR is critical for mammary cancer growth, and that IGF-IR may be an upstream regulator of ErbB1/2 action. The accumulation of in vitro and in vivo evidence implicating IGF-IR as a therapeutic target will be tested in the next 5 years with the clinical advancement of various strategies to target this receptor. It is hoped that this endeavour will be fruitful and will provide a new weapon in the fight against breast cancer.

\section{References}

1. LeRoith $D$, Roberts Jr CT. The insulin-like growth factor system and cancer. Cancer Lett 2003; 195: 127-137.

2. Baserga R. The insulin-like growth factor I receptor: a key to tumor growth? Cancer Res 1995; 55: 249-252.

3. Surmacz E. Growth factor receptors as therapeutic targets: strategies to inhibit the insulin-like growth factor I receptor. Oncogene 2003; 22: 6589-6597.

4. Balana ME, Labriola $L$, Salatino $M$, et al. Activation of ErbB-2 via a hierarchical interaction between ErbB-2 and type I insulin-like growth factor receptor in mammary tumor cells. Oncogene 2001; 20: 34-47.

5. Balana ME, Lupu R, Labriola L, Charreau EH, Elizalde PV. Interactions between progestins and heregulin (HRG) signalling pathways: HRG acts as mediator of progestins proliferative effects in mouse mammary adenocarcinomas. Oncogene 1999; 18: 6370-6379.

6. Elizalde PV, Lanari C, Molinolo AA, et al. Involvement of insulin-like growth factors-I and -II and their receptors in medroxyprogesterone acetate-induced growth of mouse mammary adenocarcinomas. J Steroid Biochem Mol Biol 1998; 67: 305-317.

7. Salatino M, Schillaci R, Proietti CJ, et al. Inhibition of in vivo breast cancer growth by antisense oligodeoxynucleotides to type I insulin-like growth factor receptor mRNA involves inactivation of ErbBs, PI-3K/Akt and p42/p44 MAPK signaling pathways but not modulation of progesterone receptor activity. Oncogene 2004; 23 : 5161-5174.

8. Lee AV, Schiff R, Cui X, et al. New mechanisms of signal transduction inhibitor action: receptor tyrosine kinase down-regulation and blockade of signal transactivation. Clin Cancer Res 2003; 9: 516S-523S.

9. Coppola D, Ferber A, Miura M, et al. A functional insulinlike growth factor I receptor is required for the mitogenic and transforming activities of the epidermal growth factor receptor. Moll Cell Biol 1994; 14: 4588-4595.

10. Chakravarti A, Loeffler JS, Dyson NJ. Insulin-like growth factor receptor I mediates resistance to anti-epidermal growth factor receptor therapy in primary human glioblastoma cells through continued activation of phosphoinositide 3-kinase signaling. Cancer Res 2002; 62 200-207.

11. Lu Y, Zi X, Zhao Y, et al. Insulin-like growth factor-I receptor signaling and resistance to trastuzumab (Herceptin). J Natl Cancer Inst 2001; 93: 1852-1857.

12. Lu D, Zhang $H$, Ludwig D, et al. Simultaneous blockade of both the epidermal growth factor receptor and the insulin-like growth factor receptor signaling pathways in cancer cells with a fully human recombinant bispecific antibody. J Biol Chem 2004; 279: 2856-2865.

13. Ismail PM, Amato P, Soyal SM, et al. Progesterone involvement in breast development and tumorigenesis as revealed by progesterone receptor 'knockout' and 'knockin' mouse models. Steroids 2003; 68: 779-787. 